\title{
The furan approach to the synthesis of natural products having highly substituted cyclic ether moieties
}

\section{Abstract}

We describe the synthesis of useful building blocks towards the synthesis of many natural products bearing substituted cyclic ether moieties, using our previously described method we called "The Furan Approach" and which is based on singlet oxygen oxidation of a furan ring followed by an intramolecular oxa-Michael addition.

Keywords: oxacyclic compounds, furan, singlet oxygen, natural products, stereoselective synthesis
Volume I Issue 5 - 2017

\author{
Alioune Fall,' Ousmane Diouf,' Mohamed \\ Gaye, ' Abdou Salam Sall,' Generosa Gómez,' \\ Yagamare Fall ${ }^{2}$ \\ 'Département de Chimie, Université Cheikh Anta Diop de \\ Dakar, Sénégal \\ ${ }^{2}$ Departamento de Química Orgánica, University of Vigo, Spain
}

Correspondence: Yagamare Fall, Departamento de Química Orgánica, Facultad de Química and Instituto de Investigación Sanitaria Galicia Sur (IISGS), University of Vigo, Campus Lagoas de Marcosende, 363I0 Vigo, Spain, Tel 34 9868I2320, Email yagamare@uvigo.es Alioune Fall, Laboratoire de Chimie de Coordination Organique (LCCO), Département de Chimie, Faculté des Sciences et Techniques, Université Cheikh Anta Diop de Dakar, Sénégal,Tel 772644472,Email elhadji.fall@ucad.edu.sn

Received: October 31, 2017| Published: November 14, 2017

\section{Introduction}

Highly substituted cyclic ethers occur in many natural products exhibiting important biological activities. ${ }^{1-6}$ These units can be found in monocyclic as well as in polycyclic structures some of them are depicted in Figure 1. Due to the biological importance of these compounds, they have been the targets of numerous synthetic studies..$^{7-13}$ In our research group we have developed a new methodology for the synthesis of oxacyclic compounds using furan as starting material. ${ }^{14-27}$ The scope and limitations of this very powerful

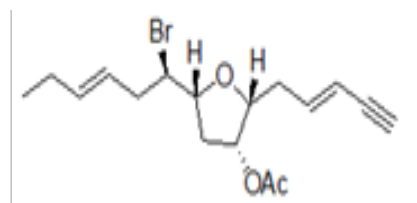

(+)-traas-kamauşne<smiles>CC(=O)CC1O[C@@H](C)CC[C@@H]1O</smiles>

(+)-Decarestrictin L methodology are being determined.

\section{Results and discussion}

We now report that using our method we can easily access the 2,6-disubstituted tetrahydropyran and 2,5-disubstituted tetrahydrofuran systems, which are advanced model intermediates towards the synthesis of the natural compounds depicted in Figure 1. It was anticipated that butanetriol (1) could be a common starting material for accessing the 2,6-disubstituted tetrahydropyran 13 (Figure 2) and 2,5-disubstituted tetrahydrofuran 22 (Figure 3).<smiles>CN(CCC#CC1CCC(O)C1COc1ccc(F)cc1)C(N)=O</smiles>

CMI-977 (LDP-977) ÓH<smiles>C[C@@H]1O[C@@H](C[N+](C)(C)C)CC1O</smiles>

(+)-Muscarine

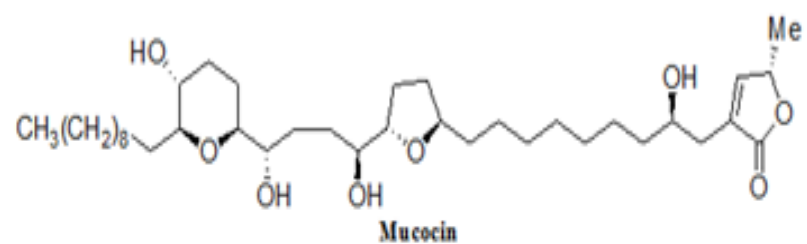

Figure I Examples of biologically active natural products bearing substituted cyclic ether moieties. 

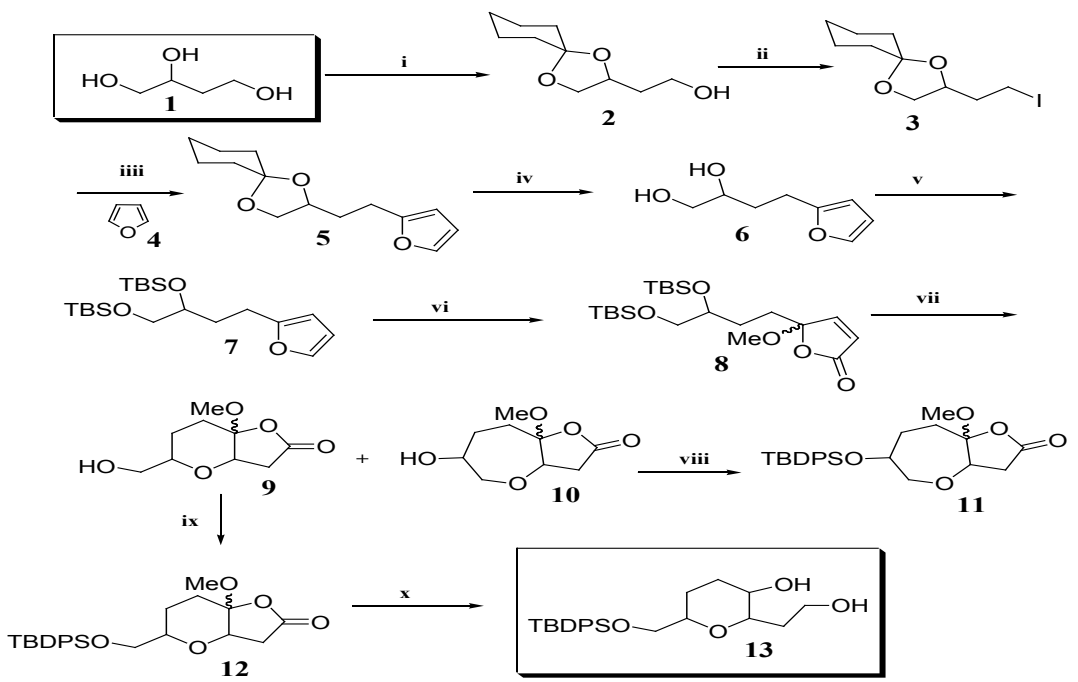

Figure 2 Reagents and conditions:

(i) Cyclohexanone, $\mathrm{BF}_{3} \cdot \mathrm{OEt}_{2}, \mathrm{Et}_{2} \mathrm{O}, 0^{\circ} \mathrm{C}$ to $\mathrm{rt}(97 \%)$

(ii) $\mathrm{PPh}_{3}$, Imid, $\mathrm{I}_{2}, \mathrm{THF}(93 \%)$

(iii) 4 , bipy, nBuLi, THF, $0^{\circ} \mathrm{C}$ to $\mathrm{rt}(91 \%)$

(iv) Dowex 50W-X8, MeOH, rt, 20h (89\%)

(v) TBSCl, Imid, DMAP, DMF, rt(97 \%)

(vi) ' $\mathrm{O}_{2}, \mathrm{MeOH}$, rose Bengal, hv

(vii) $\mathrm{Ac}_{2} \mathrm{O}$, py, DMAP (97\%\%, 2 steps)

(viii) TBAF, THF, rt $(35 \%, 9 ; 45 \%, 10)$

(ix) TBDPSCl, Imid, DMAP, DMF, rt (92\%)

(x) TBDPSCl, Imid, DMAP, DMF, rt (87 \%)

(xi) $\mathrm{LAH}_{1} \mathrm{BF}_{3} \cdot \mathrm{OEt}_{2}(86 \%)$

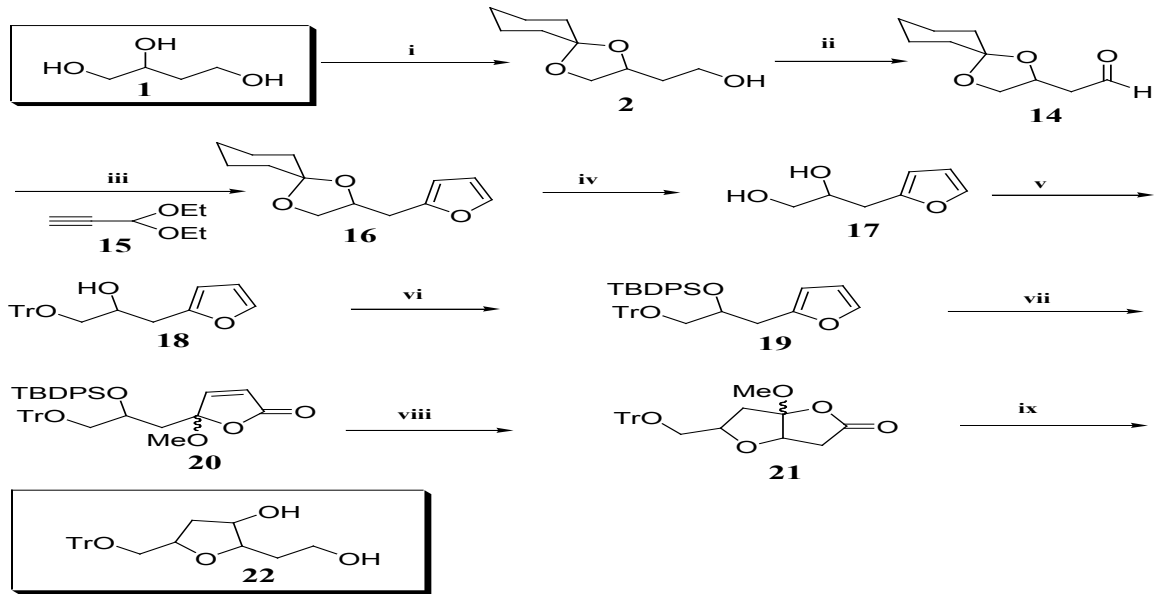

Figure 3 Reagents and conditions:

(i) Cyclohexanone, $\mathrm{BF}_{3} . \mathrm{OEt}_{2}, \mathrm{Et}_{2} \mathrm{O}, 0^{\circ} \mathrm{C}$ to $\mathrm{rt}(97 \%)$

(ii) Swern (99\%)

(iii) $15, \mathrm{nBuLi}, \mathrm{THF}, 0^{\circ} \mathrm{C}$ to $-78{ }^{\circ} \mathrm{C}(9 / \%)$

(iv) $\mathrm{H}_{2} /$ Pd Lindlar

(v) PPTs (66\%)

(vi) Dowex 50W-X8, MeOH, rt, $20 \mathrm{~h}(80 \%)$

(vii) $\mathrm{TrCl}$, pyr, DMAP, DMF, rt (82 \%)

(viii)TBDPSCl, Imid, DMAP, DMF, rt (85 \%)

(ix) ' $\mathrm{O}_{2}, \mathrm{MeOH}$, rose Bengal, hv

(x) $\mathrm{Ac}_{2} \mathrm{O}, \mathrm{py}, \mathrm{DMAP}(77 \% \%, 2$ steps)

(xi) TBAF,THF, rt (98\%)

(xii) $\mathrm{LAH}_{3} \mathrm{BF}_{3} . \mathrm{OEt}_{2}(86 \%)$

Citation: Fall A, Diouf O, Gaye M, et al.The furan approach to the synthesis of natural products having highly substituted cyclic ether moieties. MOJ Biorg Org Chem. 20I7; I (5):I84-I87. DOI: I0.I5406/mojboc.2017.0I.00033 
Thus protection of the $\mathrm{C} 1, \mathrm{C} 2$-hydroxyl groups of 1 with cyclohexanone afforded alcohol $2^{28}(97 \%)$ easily converted into iodide $3^{28}$ in $93 \%$ yield. Lithiation of furan 4 and reaction with 3 afforded the alkylated furan $5^{28}(91 \%)$. Removal of the cyclohexylidene group of 5 using Dowex 50W-X8 in methanol, ${ }^{29}$ gave diol $6^{28}$ in $89 \%$ yield. The hydroxyl groups of 6 were protected as silylethers affording furan $7 .{ }^{28}$ Oxidation of 7 with singlet oxygen followed by treatment with acetic anhydride in pyridine, afforded butenolide $8^{28}$ in $97 \%$ yields ( 2 steps). Treatment of 8 with TBAF led to lactones $9^{28}$ and $10^{28}$ in 35 and $45 \%$ yield respectively.

Protection of the hydroxyl group of 9 followed by opening of the lactone ring afforded tetrahydropyran $13^{28}$ possible synthon towards the synthesis of decarestrictine L or mucocin (Figure 1). Having demonstrated the feasibility of highly substituted tetrahydropyrans from butanetriol (1), we now turned our attention to synthesizing tetrahydrofurans from the same starting material. In order to apply our method, we needed to prepare furan 19 (Figure 3) which has a side chain one carbon shorter than its homologue ${ }^{30-31}$ (Figure 2). Swern oxidation of alcohol 2 afforded aldehyde $14^{28}$ in $99 \%$ yields.

Treatment of aldehyde 14 with the lithium derivative of alkyne 15 gave a mixture of epimeric propynyl alcohols which were hydrogenated over lindlar catalyst ${ }^{30-31}$ to provide a mixture of diastereoisomeric (Z)-alkenes which upon treatment with catalytic pyridinium toluene -p- sulfonate (PPTS) gave the desired furan ring in $66 \%$ overall yield from the aldehyde 14 . Removal of the cyclohexylidene group of $16^{28}$ using Dowex 50W-X8 in methanol ${ }^{29}$ gave diol $17^{28}$ in $80 \%$ yield. The primary hydroxyl group of 17 was selectively protected as trityl ether and the secondary hydroxyl group as silylether affording furan $19 .{ }^{28}$ The stage was now set for the oxidation of 19 with singlet oxygen followed by treatment with acetic anhydride in pyridine which afforded butenolide $20^{28}$ in $77 \%$ overall yield. Treatment of 20 with TBAF led to lactone $21^{28}$ in $98 \%$ yields. Finally, opening of lactone 21 with LAH afforded tetrahydrofuran $22 .{ }^{28}$ Compound 22 can be considered as building block towards the synthesis of the natural products bearing a THF moiety depicted in Figure 1.

\section{Conclusion}

In conclusion, we have demonstrated that using racemic butanetriol we can apply the "Furan approach "to the synthesis of useful building blocks towards the synthesis of many natural products. As both enantiomers of butanetriol are commercially available, work is now in progress towards the enantioselective synthesis of the natural products described in Figure 1.

\section{Acknowledgements}

The work of the NMR and MS divisions of the research support services of the University of Vigo (CACTI) is gratefully acknowledged. Alioune Fall, Ousmane Diouf, Abdou Salam Sall and Mohamed Gaye thank the University Cheikh Anta Diop (Dakar) for financial support.

\section{Conflict of interest}

The author declares no conflict of interest.

\section{References}

1. Kang EJ, Lee E. Total synthesis of oxacyclic macrodiolide natural products. Chem Rev. 2005;105(12):4348-4378.

2. Nakata T. Total synthesis of marine polycyclic ethers. Chem Rev. 2005;105(12):4314-4347.
3. Álvarez E, Candenas ML, Pérez R, et al. Useful Designs in the Synthesis of Trans-Fused Polyether Toxins. Chem Rev. 1995;95(6):1953-1980.

4. Shimizu YM. Microalgal metabolites. Chem Rev. 1993;93:1685-1698.

5. Yasumoto T, Murata M. Marine toxins. Chem Rev. 1993;93(5):18971909.

6. Boukouvalas J, Radu II. A concise asymmetric synthesis of $(+)-$ muscarine from (S)- $\gamma$-hydroxymethyl- $\gamma$-butyrolactone. Tetrahedron lett. 2007;48(17):2971-2973.

7. Bikádi Z, Simonyi M. Muscarinic and nicotinic cholinergic agonists: structural analogies and discrepancies. Curr Med Chem. 2003;10(23):2611-2620.

8. Agrawal D, Sriramurthy V, Yadav VK. 4,5-Didehydro-7-silyloxymethyl-2-oxepanone and formal total syntheses of Hagen's gland lactones and trans-kumausynes. Tetrahedron Lett. 2006;47(43):7615-7618.

9. Chandler CL, Phillips A. A total synthesis of (+/-)-Trans-kumausyne. Org Lett. 2005;7(16):3493-3495.

10. Carreño MC, Des Mazery R, Urbano A, et al. Reductive Cyclizations of Hydroxysulfinyl Ketones: Enantioselective Access to Tetrahydropyran and Tetrahydrofuran Derivatives. J Org Chem. 2003;68(20):7779-7787.

11. Gurjar MK, Murugaiah AMS, Radhakrishna P, et al. A novel and simple asymmetric synthesis of CMI-977 (LDP-977):a potent anti-asthmatic drug lead. Tetrahedron: Asymmetry. 2003;14(10):1363-1370.

12. Crimmins MT, Zhang Y, Diaz FA. Total Synthesis of (-)-Mucocin. Org Lett. 2006;8(11):2369-2372.

13. Takahashi S, Kubota A, Nakata T. Stereoselective Total Synthesis of Mucocin, an Antitumor Agent. Angew Chem Int Ed. 2002;41(24):47514754 .

14. Fall Y, Vidal B, Alonso D, et al. Synthesis of seven-membered oxacycles. Part 2: The furan approach. Tetrahedron Lett. 2003;44(24):4467-4469.

15. Pérez M, Canoa P, Gómez G, et al. The furan approach to oxacycles: synthesis of medium-size 2,3-disubstituted oxacycles. Tetrahedron Lett. 2004;45(27):5207-5209.

16. Alonso D, Pérez M, Gómez G, et al. The furan approach to oxacycles. Part 3: Stereoselective synthesis of 2,3-disubstituted tetrahydropyrans. Tetrahedron. 2005;61(8):2021-2026.

17. Teijeira M, Suárez PL, Gómez G, et al. The furan approach to oxacycles. Part 5: Synthesis of a chiral butenolide, building block towards biologically interesting natural products. Tetrahedron Lett. 2005;46(35):58895892 .

18. García I, Gómez G, Teijeira M, et al. The furan approach to oxacycles. Part 4: A synthesis of (+)-decarestrictine L. Tetrahedron Lett. 2006;47(8):1333-1335.

19. Canoa P, Pérez M, Covelo B, et al. The furan approach to oxacycles. Part 6: From THF to fused polyoxepanes. Tetrahedron Lett. 2007;48(19):3441-3443.

20. Álvarez C, Pérez M, Zúñiga A, et al. Synthesis of Enantiomerically Pure 2,5-Disubstituted 3-Oxygenated Tetrahydrofurans. Synthesis. 2010;22:3883-3890.

21. Canoa P, Gándara Z, Pérez M, et al. The Furan Approach to Oxacycles: New Entry to the Synthesis of Isodideoxynucleosides. Synthesis. 2011;3:431-436.

22. Gónzalez M, Gándara Z, Covelo B, et al. Total synthesis of (-)-muricatacin. Tetrahedron Lett. 2011;52(45):5983-5986.

23. Zúñiga A, Pazos G, Besada P, et al. Synthesis of a Greek tobacco lactonic natural product and its analogues. Tetrahedron Lett. 2012;53(33):42934295 . 
24. González M, Gándara Z, Pazos G, et al. Synthesis of (-)-Muricatacin from Tri-O-acetyl-d-glucal. Synthesis. 2013;45:625-632.

25. Gónzalez M, Gándara Z, Martínez A, et al. Synthesis of (4S, 5S, 11R) and (4S, 5S, 11S)-iso-cladospolide B. Tetrahedron Lett. 2013;54(28):36473650 .

26. Gónzalez M, Gándara Z, Martínez A, et al. Synthesis of (+)-Muricatacin and a Formal Synthesis of CMI-977 from 1-Malic Acid. Synthesis. 2013;45(12):1693-1700.

27. González M, Gándara Z, Seck M, et al. First total synthesis of (4R,5R,11S) and (4R,5R,11R)-iso-cladospolide B. Mediterr J Chem. 2015;4(1):18-29.
28. All new compounds exhibited satisfactory $1 \mathrm{H}$ and $13 \mathrm{C}$ NMR, analytical, and/or high resolution mass spectral data.

29. López S, Fernández-Trillo F, Midón P, et al. First Stereoselective Syntheses of (-)-Siphonodiol and (-)-Tetrahydrosiphonodiol, Bioactive Polyacetylenes from Marine Sponges. J Org Chem. 2005;70(16):63466352.

30. Cram DJ, Allinger NL. Macro Rings. XIII. Synthesis and Properties of 1,7-Cyclododecadiyne and Related Compounds ${ }^{1}$. J Am Chem Soc. 1956;78(11):2518-2524.

31. Kocienski PJ, Brown RCD, Pommier A, et al. Synthesis of salinomycin. J Chem Soc Perkin Trans. 1998;1:9-40. 